\title{
RF BASED SIGN BOARD DETECTION AND COLLISION AVOIDANCE SYSTEM
}

\author{
Ravikiran Nayak $^{1 *}$, Dilip Chandra ${ }^{2}$ \\ *1,2 School of Electronic and Communication Engineering REVA UNIVERSITY, Bangalore \\ *1Email: ravi.rtn94@gmail.com, ${ }^{2}$ Email: dilipe@ revainstitution.org
}

*Corresponding Author: -

Email: ravi.rtn94@gmail.com

\begin{abstract}
: -
Nowadays the percentage of accidents has been going up day by day. This might be because of driver negligence of not following the traffic rules and sign boards. RF based sign board detection and collision avoidance system is such approach where the rider of the vehicle will get advance intimation of fore coming hurdles. Also, it detects front vehicle distance. This system is very helpful in cloudy area, curved roads. At night it is very difficult for driver to see the sign boards, answer for these issues is the 'RF based sign board detection and collision avoidance system'.
\end{abstract}

Keyword: Arduino AT mega 2560, speed control, $R F$ 


\section{I.INTRODUCTION}

This project is based on Arduino mega 2560 microcontroller which is an 8-bit controller. The transmitter will be placed in the sign boards and the receiver will be placed in the vehicle. Whenever the vehicle enters into transmitter range it'll get connected to the transmitter and corresponding code for that sign board will be sent to the receiver. The data is sent wirelessly using RF based wireless transmission [1]. The received data will be displayed on to LCD display and also on the Android phone.

The ultrasonic sensor will be placed in front of the vehicle. It will measure the distance of the front vehicle, if it is in the critical zone the controller will halt the vehicle and intimate the driver.

Driver will be already using so many controllers in the vehicle it is very difficult to keep track of different sign boards such as school zone, speed limit, curved road, intersections and other sign related information. But these sign boards are usually not placed properly. In its place we send indication to the driver about upcoming signs from a distant area and the vehicle will receive this information before reaching that area wirelessly then prior action can be taken in order to avoid the misfortunes.

The module has 2 parts

1)RF Transmitter-which has an Encoder, which is used to transmit the 4-bit parallel data serially. 2)Controller with RF receiver-which has a Decoder, android, display and other indication devices.

\section{LITERATURE SURVEY}

Road accidents are now globally recognized as a serious public health problem. The problem is much more serious in our country where close to 5,00,000 road accidents caused nearly 1,46,000 deaths and left more than thrice that number injured. Collision warning and collision avoidance systems are emerging automotive safety technologies that assist drivers in avoiding rear-end collisions. Their function is to allow the driver enough time to avoid the crash and yet avoid annoying the driver with alerts perceived as occurring too early or unnecessary. A prime example of driver assistance systems is cruise control, which has the capability of maintaining a constant user pre-set speed and its evolution, the adaptive cruise control, which adds to cruise control the capability of keeping a safe distance from the preceding vehicle. A drawback of these systems is that they are not independently capable of distinguishing between straight and curved parts of the road, where the speed has to be lowered to avoid accidents.

Many of the traffic communicators are present in the market that functions as initializing a vehicle wireless subsystem; broadcasting a query to request real-time traffic pattern data, where in the real-time traffic pattern data comprises traffic pattern data obtained from other vehicle wireless subsystems and highway wireless subsystems; and if a response to the query has been received, incorporating the real-time traffic pattern data in to a runtime database and creating a humanreadable display for displaying on a navigation system [2]. For all these processes to be done the vehicle must have a GPRS \& computer system or any real time operating system and to make that system run effectively another by another person rather than driver has to be, which is not economical and difficult to implement on all the speed vehicles on highways.

However, these all-traditional safety systems are in post-accident action to protect driver and passengers. But these systems do not avoid the probability of accidents. Thus, we need a driver assistance system to make vehicle stop in a safety zone if it detects the probability of accident. Also, these systems are programmed to assist driver for breaking, acceleration and maintain a safe distance from vehicle ahead.

A collision avoidance system operates, generally, in the following manner: a sensor installed at the front-end of a vehicle constantly scans the road ahead for vehicles or obstacles. When found, the system determines whether the vehicle is in imminent danger of crashing, and if so, automatic brakes should be applied.

\section{III.PROPOSED BLOCK DIADRAM}

In this project the speed control of DC motor is done via L298N motor driver, which consist of dual full $\mathrm{H}$-bridge to control 2 motors. The Arduino mega 2560 microcontroller is used to design the sign board detection; the programming is done using Arduino software in Embedded c language. 


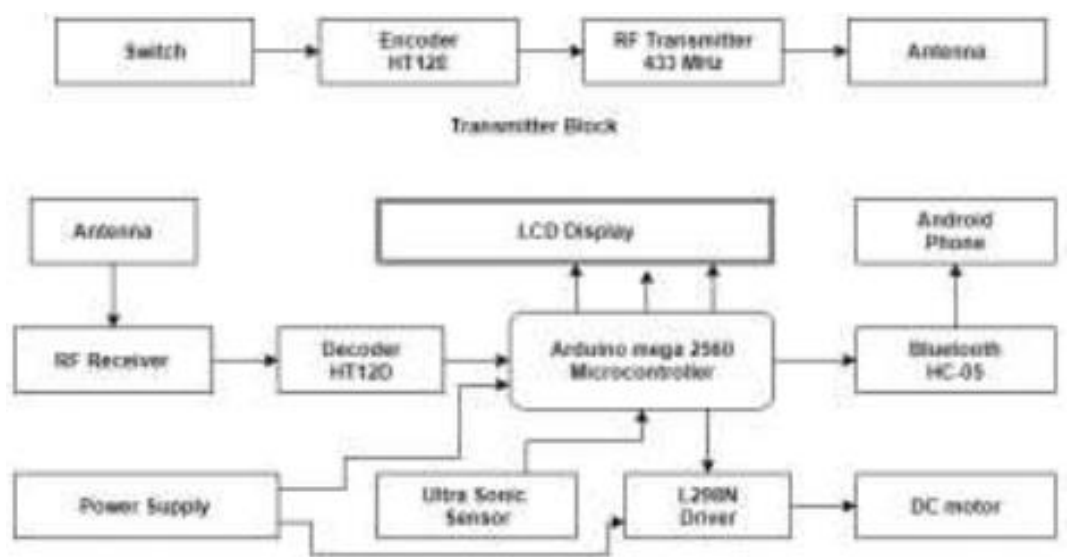

Fig 1. Proposed block diagram

The Figure 1 shows the block diagram of proposed system. The receiver block mainly consists of Arduino ATmega 2560 microcontroller which is heart of this system, which controls the whole system. It has $256 \mathrm{~KB}$ of Flash memory for storing code out of which $8 \mathrm{~KB}$ used by bootloader, $8 \mathrm{~KB}$ of SRAM, and $4 \mathrm{~KB}$ of EEPROM and runs at clock speed of $16 \mathrm{MHz}$, and has 54 digital pins out of which 15 provides PWM output, 16 are analog inputs and 10 pins for communication. The transmitter block as shown in block diagram it consists of switch which is used to send particular code through Encoder which transmits the 12-bit parallel data into serially. In the receiver block ATmega 2460 controller is the heart of the system as it controls the whole system. The code is received through RF receiver which is connected to Decoder, which decodes the 12- bit serial data into parallel. The received data is displayed onto LCD display and also in the Android phone which is connected to controller via HC-05 Bluetooth.

The Ultrasonic sensor is used to measure the distance of the front vehicle. The code is written in a such way that it'll have 3 stages of indication i.e., critical zone, normal zone and mid-range. When the vehicle enters into critical zone alert message is sent to Android and the vehicle becomes halt. In other zones the controller will continuously send distance of the vehicle to the driver i.e it just gives indication to the driver.

Fig 2. showas the flow of the speed control of the DC motor. Initially the DC motor is connected to L298N Driver which has dual H-bridge. The input pins and enable pin from the Driver is connected to the ATmega 2560 microcontroller.

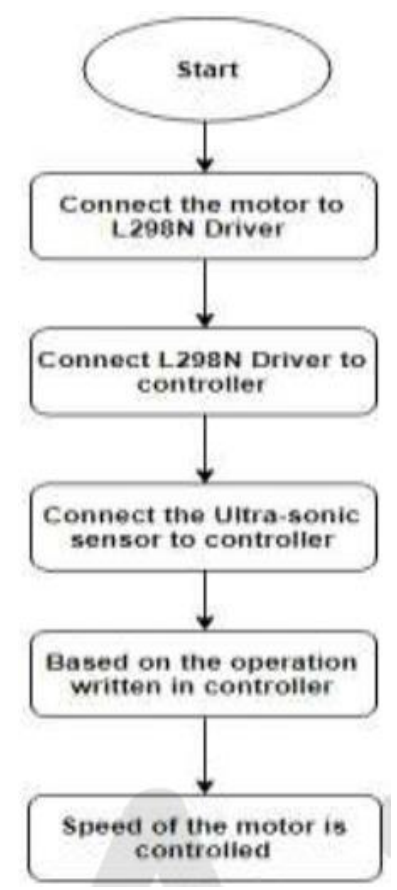

Fig 2. flow chart motor speed control

Ultra-sonic sensor will also play an important role in the speed control of DC motor. The sensor has four pins Echo, Trigger, Gnd and Vcc. It works on the principle of Pizeo-electric effect. It has two main parts i.e a transmitter and a receiver, the transmitter will generate ultrasonic waves when these waves hit an object the reflected wave will collected in the receiver. It can used for many other applications also such as, level detector, Intruder alarm system and distance measuring etc.

As I told earlier it will have 3 stages, when it reaches the critical zone the speed of the motor is controlled by the ATmega 2560 microcontroller. 


\section{IV.IMPLEMENTATION}

The Android App is going to be written using MIT app inventor which is not yet implemented, but in future it is going to be implemented. It is an visual, Block language for building app. The codes will shown in the form of blocks which is easy to understand.

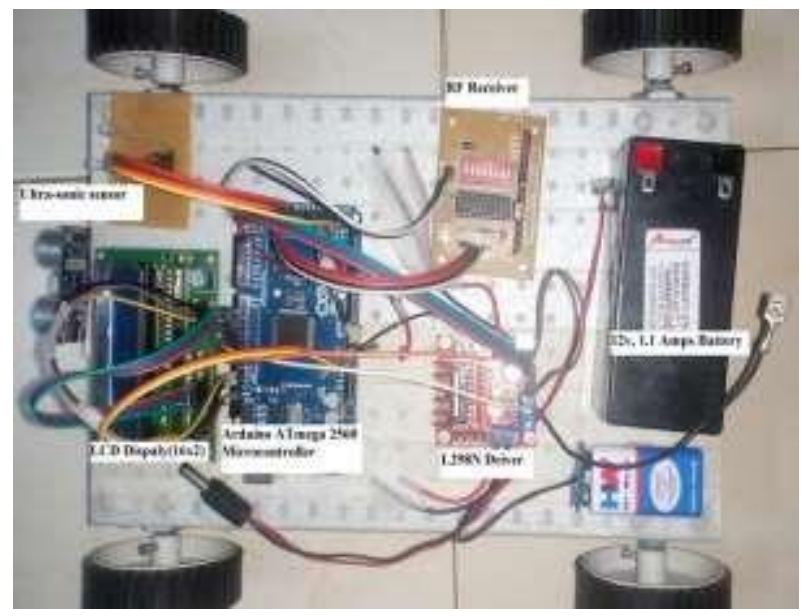

Fig 3. Experimental Hardware

The whole receiver block is placed onto the vehicle which is running using $2300 \mathrm{rpm}$ DC motors. $12 \mathrm{v}$ battery is used to provide supply to these motors. The power is supplied to Transmitter block using 9v battery. Each of these Switches contains the particular code of sign boards.

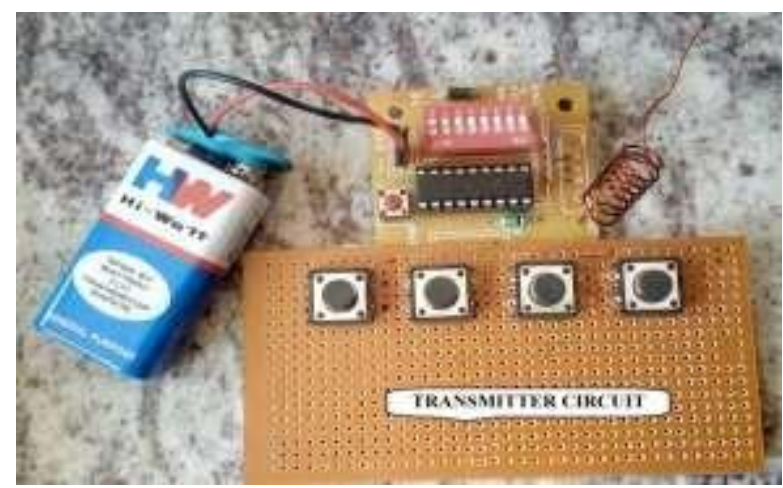

Fig 4. Transmitter block

For demo I have made the vehicle to run or move at normal speed and when I send signal from the Transmitter any one out of four based on that the operations is performed at receiver block. For example if I send Speed limit signal to the receiver block then the speed of the motor is reduced and sign board details will be shown in the Display. As shown in the fig 5 .

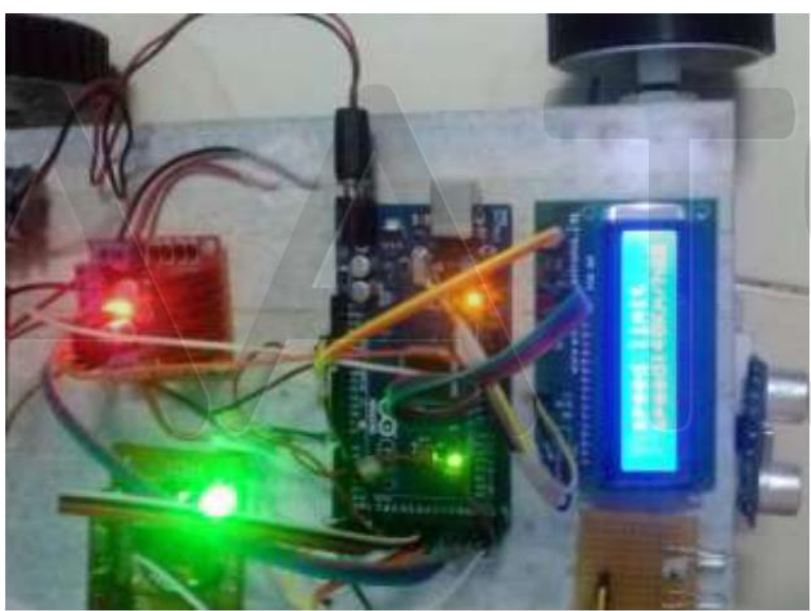

Fig 5.output showing onto LCD

\section{CONCLUSION}

The implemented project will helps to driver by letting him know what's coming ahead. By using this no of accidents 
can be reduced and follow the sign boards. Vehicle also stops when the any vehicle or object is in critical zone of the vehicle. In future it can help to reduce accidents in greater extent as most of the accidents take place when signboards are avoided.

\section{REFERENCES}

[1].Ankita Mishra 1, Jyoti Solanki 2 , Harshala Bakshi 3, Priyanka Saxena 4, Pranav Paranjpe —Design of RF based speed control system for vehicles\| International Journal of Advanced Research in Computer and Communication Engineering, Vol. 1, Issue 8, October 2012.

[2].Telaprolu.m.k,sarma,V.V.;:;ratankanth,E.K.;Rao,S. N.;Banda,v.,vehicular Electronics and safety (ICVES), IEEE international conference Pune (2009).

[3].Kassem, N. Microsoft Corp., Redmond, WA, USA Kosba, A.E.; Youssef, M.;VRF-Based Vehicle Detection and Speed Estimation vehicular Technology Conference (VTC Spring), IEEE (2012). 